\title{
Worst-Case Robust Multiuser Transmit Beamforming Using Semidefinite Relaxation: Duality and Implications
}

\author{
Tsung-Hui Chang ${ }^{\star}$, Wing-Kin $\mathrm{Ma}^{\dagger}$, and Chong-Yung Chi ${ }^{\star}$ \\ ${ }^{\star}$ Institute of Commun. Eng. \& Dept. of Elect. Eng. \\ National Tsing Hua University, \\ Hsinchu, Taiwan 30013 \\ E-mail: tsunghui.chang@ieee.org, cychi@ee.nthu.edu.tw \\ ${ }^{\dagger}$ Department of Electronic Engineering \\ The Chinese University of Hong Kong, \\ Shatin, N.T., Hong Kong \\ E-mail: wkma@ieee.org
}

\begin{abstract}
This paper studies a downlink multiuser transmit beamforming design under spherical channel uncertainties, using a worst-case robust formulation. This robust design problem is nonconvex. Recently, a convex approximation formulation based on semidefinite relaxation (SDR) has been proposed to handle the problem. Curiously, simulation results have consistently indicated that SDR can attain the global optimum of the robust design problem. This paper intends to provide some theoretical insights into this important empirical finding. Our main result is a dual representation of the SDR formulation, which reveals an interesting linkage to a different robust design problem, and the possibility of SDR optimality.
\end{abstract}

\section{INTRODUCTION}

This paper focuses on a standard wireless multiuser unicast system where a multiple-antenna transmitter broadcasts independent data streams to multiple single-antenna receivers using transmit beamforming [1]. In this context, the efficacy of beamforming designs relies on knowledge of the channel state information (CSI) of all the receivers. However, the transmitter often has some uncertainties on the CSI, due to issues such as finite-length training and finite-rate feedback [2]. CSI uncertainties at the transmitter can result in significant performance outage, if not taken into consideration in the beamforming designs. The CSI uncertainty problem has motivated considerable research endeavors in robust transmit beamforming design techniques. This includes the chance constrained robust designs [3], [4], where the CSI uncertainties are modeled as random variables, and the worst-case robust designs [5]-[8], where the CSI uncertainties are modeled as bounded unknowns within a predetermined, small error set.

Our problem of interest is the worst-case signal-tointerference-plus-noise ratio (SINR) constrained robust transmit beamforming design problem under spherically bounded CSI uncertainties, which has drawn much interest recently [6][8]. Presently available beamforming solutions for this worstcase robust problem are based on approximation methods, either restriction [6], [7] or relaxation [8], and it is now not clear whether the worst-case robust problem can be optimally (and efficiently) solved. However, simulations seem to have provided the answer to the latter- the semidefinite relaxation (SDR) method [8]. SDR is a convex relaxation technique for a certain class of hard (nonconvex) optimization problems, and has recently gained popularity owing to its wide scope of applicability [9], [10]. For a general application, SDR is considered a suboptimal solver; however, for the worstcase robust beamforming problem, simulation results have indicated that SDR should to be a globally optimal solver, which is a rather surprising empirical finding. As such, being able to provide a theoretical analysis proving whether SDR is optimal would be of much significance. A recent result [11] has partially addressed this open question, where the SDR optimality under sufficiently small error radii is analyzed.

This paper intends to address the mystery of SDR optimality in worst-case robust transmit beamforming optimization using a different analysis approach. We show that the worst-case robust problem has a close relationship to a different robust beamforming problem, in form of max-min optimization. In particular, we prove that their SDR problems are dual, or equivalent, to each other. This new, intriguing, duality relationship provides a new perspective and useful insights explaining the optimality of SDR. In particular, we will give a condition under which SDR provides globally optimal solutions to the worst-case robust problem.

\section{SignAl Model AND BACKGROUND}

Consider a wireless downlink system where a transmitter, equipped with $N_{t}$ antennas, wants to communicate with $K$ single-antenna receivers using transmit beamforming. The problem formulation follows a standard unicast setting [1]: Let $\boldsymbol{h}_{i} \in \mathbb{C}^{N_{t}}$ denote the channel vector of receiver $i$, and let $\boldsymbol{w}_{i} \in \mathbb{C}^{N_{t}}$ be the associated beamforming vector for receiver $i$. The SINR of receiver $i$ is given by

$$
\operatorname{SINR}_{i}\left(\boldsymbol{w}_{1}, \ldots, \boldsymbol{w}_{K}, \boldsymbol{h}_{i}\right)=\frac{\left|\boldsymbol{h}_{i}^{H} \boldsymbol{w}_{i}\right|^{2}}{\sum_{k \neq i}^{K}\left|\boldsymbol{h}_{i}^{H} \boldsymbol{w}_{k}\right|^{2}+\sigma_{i}^{2}},
$$

where $\sigma_{i}^{2}>0$ is the noise power at receiver $i$, for all $i=1, \ldots, K$. Our goal is to design the beamforming vectors $\left\{\boldsymbol{w}_{i}\right\}_{i=1}^{K}$ such that each receiver achieves a desired SINR level.

Conventionally, transmit beamforming designs require full channel state information (CSI) at the transmitter; i.e., knowledge of $\left\{\boldsymbol{h}_{i}\right\}_{i=1}^{K}$. In wireless communications, however, it 
is difficult for the transmitter to acquire accurate CSI, due to imperfect channel estimation and finite rate feedback [2]. Hence there are channel uncertainties at the transmitter; i.e.,

$$
\boldsymbol{h}_{i}=\overline{\boldsymbol{h}}_{i}+\boldsymbol{e}_{i}, i=1, \ldots, K,
$$

where $\overline{\boldsymbol{h}}_{i}$ denotes the channel estimate available at the transmitter, and $\boldsymbol{e}_{i} \in \mathbb{C}^{N_{t}}$ represents the channel uncertainty. In this work, we focus on spherically bounded channel uncertainties:

$$
\left\|\boldsymbol{e}_{i}\right\|^{2} \leq r_{i}^{2}, i=1, \ldots, K
$$

where $\|\cdot\|$ denotes the Euclidean norm, and $r_{i}>0$ is the radius of the uncertainty ball. We study the following worstcase robust beamforming design [6], [8]:

$$
\begin{aligned}
\min _{\substack{\boldsymbol{w}_{i} \in \mathbb{C}^{N_{t}}, i=1, \ldots, K}} & \sum_{i=1}^{K}\left\|\boldsymbol{w}_{i}\right\|^{2} \\
\text { s.t. } & \operatorname{SINR}_{i}\left(\boldsymbol{w}_{1}, \ldots, \boldsymbol{w}_{K}, \overline{\boldsymbol{h}}_{i}+\boldsymbol{e}_{i}\right) \geq \gamma_{i} \forall\left\|\boldsymbol{e}_{i}\right\|^{2} \leq r_{i}^{2}, \\
& i=1, \ldots, K,
\end{aligned}
$$

where $\gamma_{i}>0$ is the SINR requirement of receiver $i$, which must be fulfilled even under worst possible CSI uncertainties.

The challenge of solving the worst-case robust problem (4) lies in the worst-case SINR constraints in (4b), each of which corresponds to an infinite number of nonconvex quadratic constraints. As mentioned, there are several approximation methods for managing problem (4) [6]-[8], and here we focus on the SDR method [8]. The development of SDR consists of two steps. The first step, which is standard (see, e.g. [10]), is to substitute $\boldsymbol{W}_{i}=\boldsymbol{w}_{i} \boldsymbol{w}_{i}^{H}, k=1, \ldots, K$, into (4b), and then replace $\boldsymbol{W}_{i}=\boldsymbol{w}_{i} \boldsymbol{w}_{i}^{H}$ by $\boldsymbol{W}_{i} \succeq \mathbf{0}$ (i.e., $\boldsymbol{W}_{i}$ being positive semidefinite (PSD)) to obtain a relaxed problem

$$
\begin{aligned}
\min _{\substack{\boldsymbol{W}_{i} \in \mathbb{H}^{N_{t}}, i=1, \ldots, K}} & \sum_{i=1}^{K} \operatorname{Tr}\left(\boldsymbol{W}_{i}\right) \\
\text { s.t. } & \left(\overline{\boldsymbol{h}}_{i}+\boldsymbol{e}_{i}\right)^{H}\left(\frac{1}{\gamma_{i}} \boldsymbol{W}_{i}-\sum_{k \neq i}^{K} \boldsymbol{W}_{k}\right)\left(\overline{\boldsymbol{h}}_{i}+\boldsymbol{e}_{i}\right) \geq \sigma_{i}^{2} \\
& \forall\left\|\boldsymbol{e}_{i}\right\|^{2} \leq r_{i}^{2}, \quad k=1, \ldots, K \\
& \boldsymbol{W}_{1}, \ldots, \boldsymbol{W}_{K} \succeq \mathbf{0}
\end{aligned}
$$

where $\mathbb{H}^{N_{t}}$ is the set of all $N_{t}$ by $N_{t}$ Hermitian matrices, and $\operatorname{Tr}\left(\boldsymbol{W}_{i}\right)$ denotes the trace of $\boldsymbol{W}_{i}$. The motivation of this step is to linearize the nonconvex constraints. The second step is to turn (5b) to finite numbers of constraints, thereby enabling efficient implementations. By applying $\mathcal{S}$-lemma (see [12]) to (5b), we obtain the following SDR formulation of (4):

$$
\begin{aligned}
\min _{\substack{\boldsymbol{W}_{i} \in \mathbb{H}^{N_{t}}, \lambda_{i} \in \mathbb{R}, i=1 \ldots, K}} & \sum_{i=1}^{K} \operatorname{Tr}\left(\boldsymbol{W}_{i}\right) \\
\text { s.t. } & \boldsymbol{\Psi}_{i}\left(\boldsymbol{W}_{1}, \ldots, \boldsymbol{W}_{K}, \lambda_{i}\right) \succeq \mathbf{0}, i=1, \ldots, K, \\
& \boldsymbol{W}_{1}, \ldots, \boldsymbol{W}_{K} \succeq \mathbf{0}, \lambda_{1}, \ldots, \lambda_{K} \geq 0,
\end{aligned}
$$

where the matrix functions $\boldsymbol{\Psi}_{i}\left(\boldsymbol{W}_{1}, \ldots, \boldsymbol{W}_{K}, \lambda_{i}\right)$ are defined as

$$
\begin{array}{r}
\boldsymbol{\Psi}_{i}\left(\boldsymbol{W}_{1}, \ldots, \boldsymbol{W}_{K}, \lambda_{i}\right) \triangleq\left[\begin{array}{c}
\boldsymbol{I} \\
\overline{\boldsymbol{h}}_{i}^{H}
\end{array}\right]\left(\frac{1}{\gamma_{i}} \boldsymbol{W}_{i}-\sum_{k \neq i}^{K} \boldsymbol{W}_{k}\right)\left[\boldsymbol{I} \overline{\boldsymbol{h}}_{i}\right] \\
+\left[\begin{array}{cc}
\lambda_{i} \boldsymbol{I} & \mathbf{0} \\
\mathbf{0} & -\sigma_{i}^{2}-\lambda_{i} r_{i}^{2}
\end{array}\right], i=1, \ldots, K, \quad(7)
\end{array}
$$

where $\boldsymbol{I}$ is the $N_{t}$ by $N_{t}$ identity matrix. Note that the SDR problem (6) is a semidefinite program (SDP), which is convex and tractable.

The SDR problem (6) is methodologically an approximation to the worst-case robust problem (4) because the ranks of $\boldsymbol{W}_{i}$ are not constrained. However, if the optimal solution of the SDR problem (6), denoted by $\left(\boldsymbol{W}_{1}^{\star}, \ldots, \boldsymbol{W}_{K}^{\star}\right)$, is of rank one; i.e., $\boldsymbol{W}_{i}^{\star}=\boldsymbol{w}_{i}^{\star}\left(\boldsymbol{w}_{i}^{\star}\right)^{H}$ for all $i=1, \ldots, K$, then it can be verified that $\left(\boldsymbol{w}_{1}^{\star}, \ldots, \boldsymbol{w}_{K}^{\star}\right)$ is a globally optimal solution to the worst-case robust formulation (4). Rather surprisingly, it is found through simulations that SDR yields rank-one solution automatically, and it happens seemingly all the time [8], [11] (see also [4]). Our endeavor in the subsequent section is to provide a dual formulation of the SDR problem (6) that may shed light into this empirical finding.

Before we proceed to the main result, let us present some simulation results to further strengthen the motivation of the raised analysis problem. Specifically, we benchmark the SDR method against other concurrent approximation methods, namely, the robust SOCP-based method in [6], and the MMSEbased SDP method in [7]. The simulation settings are: $N_{t}=4$, $K=4, \gamma \triangleq \gamma_{1}=\cdots=\gamma_{K}, \sigma_{1}^{2}=\cdots=\sigma_{K}^{2}=0.1$, $r \triangleq r_{1}=\cdots=r_{K}=0.1$, and $\left(\overline{\boldsymbol{h}}_{1}, \ldots, \overline{\boldsymbol{h}}_{K}\right)$ being independent and identically distributed complex Gaussian random variables with zero mean and unit variance. The result is shown in Fig. 1, where we see that the SDR method outperforms the other two methods. Moreover, we should emphasize that the SDR method yielded rank-one solution in all the trials ran.

III. Duality of Worst-Case Robust SDR

Consider the following max-min optimization problem

$$
\begin{aligned}
\max _{\substack{\boldsymbol{e}_{i} \in \mathbb{C}^{N_{t}}, i=1, \ldots, K}}\left\{\begin{aligned}
& \min _{\substack{\boldsymbol{w}_{i} \in \mathbb{C}^{N_{t}} \\
i=1, \ldots, K}} \sum_{i=1}^{K}\left\|\boldsymbol{w}_{i}\right\|^{2} \\
& \text { s.t. } \operatorname{SINR}_{i}\left(\boldsymbol{w}_{1}, \ldots, \boldsymbol{w}_{K}, \overline{\boldsymbol{h}}_{i}+\boldsymbol{e}_{i}\right) \geq \gamma_{i}, \\
& i=1, \ldots, K,
\end{aligned}\right. \\
\text { s.t. }\left\|\boldsymbol{e}_{i}\right\|^{2} \leq r_{i}^{2}, \quad i=1, \ldots, K .
\end{aligned}
$$

At first look, problem (8) is different from the worst-case robust problem in (4). In (8), the inner minimization is a standard non-robust beamforming design problem [1] which finds the most power efficient design given a presumed CSI $\left\{\overline{\boldsymbol{h}}_{i}+\boldsymbol{e}_{i}\right\}_{i=1}^{K}$. The outer maximization, however, targets to find a "worst" set of CSI uncertainties $\left\{\boldsymbol{e}_{i}\right\}_{i=1}^{K}$ that maximizes the inner-minimum transmit power. We should also note that problem (8) has a flavor of two-player zero-sum game.

We are particularly interested in applying SDR to (8). Like SDR for the worst-case robust problem, we replace each 


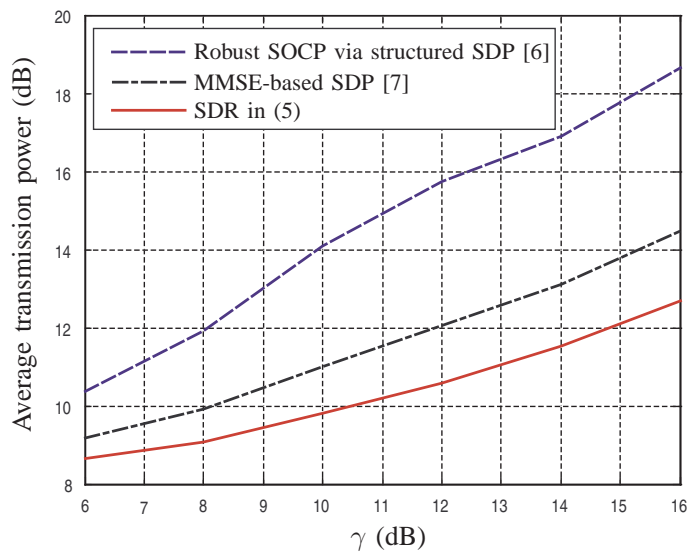

Fig. 1: Simulation results of average transmission power versus target SINR $\gamma$, for uncertainty radius $r=0.1$.

$\boldsymbol{w}_{i} \boldsymbol{w}_{i}^{H}$ with a PSD matrix $\boldsymbol{W}_{i}$, and each $\left[\boldsymbol{e}_{i}^{H} 1\right]\left[\boldsymbol{e}_{i}^{H} 1\right]^{H}$ with a PSD matrix $\boldsymbol{V}_{i}$, to obtain the following problem

$\max _{\substack{\boldsymbol{V}_{i} \in \mathbb{H}^{N_{t}+1} \\ i=1, \ldots, K}}\left\{\begin{array}{c}\min _{\boldsymbol{W}_{i} \in \mathbb{H}^{N_{t}}} \sum_{i=1}^{K} \operatorname{Tr}\left(\boldsymbol{W}_{i}\right) \\ \text { s.t. } \operatorname{Tr}\left(\left(\frac{1}{\gamma_{i}} \boldsymbol{W}_{i}-\sum_{k \neq i}^{K} \boldsymbol{W}_{k}\right) \boldsymbol{R}_{i}\right) \geq \sigma_{i}^{2}, \\ i=1, \ldots, K, \\ \boldsymbol{W}_{1}, \ldots, \boldsymbol{W}_{K} \succeq \mathbf{0} .\end{array}\right\}$ s.t. $\operatorname{Tr}\left(\boldsymbol{V}_{i}\right) \leq\left(1+r_{i}^{2}\right), i=1, \ldots, K$,

$\left[\boldsymbol{V}_{i}\right]_{N_{t}+1}=1, i=1, \ldots, K$,

$$
\boldsymbol{V}_{1}, \ldots, \boldsymbol{V}_{K} \succeq \mathbf{0},
$$

where $\left[\boldsymbol{V}_{i}\right]_{N_{t}+1}$ is the $\left(N_{t}+1, N_{t}+1\right)$ th entry of $\boldsymbol{V}_{i}$ and $\boldsymbol{R}_{i}=\left[\begin{array}{ll}\boldsymbol{I} & \overline{\boldsymbol{h}}_{i}\end{array}\right] \boldsymbol{V}_{i}\left[\begin{array}{ll}\boldsymbol{I} & \overline{\boldsymbol{h}}_{i}\end{array}\right]^{H}, i=1, \ldots, K$.

An important observation of problem (9) is that there always exists a rank-one solution for the inner minimization of problem (9):

Fact 1 [1] Consider the following SDP:

$$
\begin{aligned}
\min _{\substack{\boldsymbol{W}_{i} \in \mathbb{H}^{N_{t}}, i=1, \ldots, K}} & \sum_{i=1}^{K} \operatorname{Tr}\left(\boldsymbol{W}_{i}\right) \\
\text { s.t. } & \operatorname{Tr}\left(\left(\frac{1}{\gamma_{i}} \boldsymbol{W}_{i}-\sum_{k \neq i}^{K} \boldsymbol{W}_{k}\right) \boldsymbol{R}_{i}\right) \geq \sigma_{i}^{2}, i=1, \ldots, K, \\
& \boldsymbol{W}_{i} \succeq \mathbf{0}, i=1, \ldots, K
\end{aligned}
$$

where $\boldsymbol{R}_{1}, \ldots, \boldsymbol{R}_{K} \succeq \mathbf{0}$. Suppose that (10) is feasible. Then there exists an optimal solution $\left(\boldsymbol{W}_{1}^{\star}, \ldots, \boldsymbol{W}_{K}^{\star}\right)$ for which $\operatorname{rank}\left(\boldsymbol{W}_{i}^{\star}\right)=1$ for all $i$.

Fact 1 implies that the SDR of $\left(\boldsymbol{W}_{1}, \ldots, \boldsymbol{W}_{K}\right)$ is always tight for the max-min SDR problem (9). Fact 1 raises an intriguing question-What is the relationship between the max-min SDR problem (9) and the robust SDR problem (6)? If the optimal solutions of $\left(\boldsymbol{W}_{1}, \ldots, \boldsymbol{W}_{K}\right)$ of the two problems are identical, then Fact 1 immediately implies that (6) has a rank-one optimal solution and hence SDR is tight to (6) as well.

\section{A. Main Result}

It turns out that problems (9) and (6) are strongly connected:

Proposition 1 Suppose that problem (6) is feasible. Then problems (9) and (6) attain the same optimal objective value. Moreover, if $\left(\boldsymbol{W}_{1}^{\star}, \ldots, \boldsymbol{W}_{K}^{\star}, \lambda_{1}^{\star}, \ldots, \lambda_{K}^{\star}\right)$ is an optimal solution of problem (6), then there exists $\left(\boldsymbol{V}_{1}^{\star}, \ldots, \boldsymbol{V}_{K}^{\star}\right)$ such that $\left(\boldsymbol{V}_{1}^{\star}, \ldots, \boldsymbol{V}_{K}^{\star}, \boldsymbol{W}_{1}^{\star}, \ldots, \boldsymbol{W}_{K}^{\star}\right)$ is an outer-inner solution of problem (9).

As the main contribution of this paper, Proposition 1 provides a solution correspondence between problems (9) and (6), showing that problem (9) is actually a dual representation of problem (6). To prove that problems (9) and (6) attain the same optimal objective value, we show that the Lagrangian dual of problem (6) is equivalent to the Lagrangian dual of problem (9). The former can be shown to be

$$
\begin{aligned}
\max _{\substack{\boldsymbol{A}_{i} \in \mathbb{H}^{N_{t}+1} \\
i=1, \ldots, K}} & \sum_{i=1}^{K} \sigma_{i}^{2}\left[\boldsymbol{A}_{i}\right]_{N_{t}+1} \\
\text { s.t. } & \boldsymbol{Y}_{i}\left(\boldsymbol{A}_{1}, \ldots, \boldsymbol{A}_{K}\right) \succeq \mathbf{0}, i=1, \ldots, K, \\
& \operatorname{Tr}\left(\boldsymbol{A}_{i}\right) \leq\left(1+r_{i}^{2}\right)\left[\boldsymbol{A}_{i}\right]_{N_{t}+1}, i=1, \ldots, K, \\
& \boldsymbol{A}_{1}, \ldots, \boldsymbol{A}_{K} \succeq \mathbf{0},
\end{aligned}
$$

where $\boldsymbol{A}_{1}, \ldots, \boldsymbol{A}_{K} \in \mathbb{H}^{N_{t}+1}$ are the (Lagrangian) dual variables associated with constraints $(6 \mathrm{~b})$, and

$$
\begin{aligned}
\boldsymbol{Y}_{i}\left(\boldsymbol{A}_{1}, \ldots, \boldsymbol{A}_{K}\right) \triangleq \boldsymbol{I}-\frac{1}{\gamma_{i}}\left[\begin{array}{cc}
\boldsymbol{I} & \overline{\boldsymbol{h}}_{i}
\end{array}\right] \boldsymbol{A}_{i}\left[\begin{array}{c}
\boldsymbol{I} \\
\overline{\boldsymbol{h}}_{i}^{H}
\end{array}\right] \\
+\sum_{k=1, k \neq i}^{K}\left[\boldsymbol{I} \overline{\boldsymbol{h}}_{k}\right] \boldsymbol{A}_{k}\left[\begin{array}{c}
\boldsymbol{I} \\
\overline{\boldsymbol{h}}_{k}^{H}
\end{array}\right], i=1, \ldots, K .
\end{aligned}
$$

Now let us consider the Lagrangian dual of the inner minimization problem of (9), which can be shown to be

$$
\begin{aligned}
\max _{\mu_{1}, \ldots, \mu_{K} \geq 0} & \sum_{i=1}^{K} \mu_{i} \sigma_{i}^{2} \\
\text { s.t. } & \boldsymbol{I}-\frac{\mu_{i}}{\gamma_{i}} \boldsymbol{R}_{i}+\sum_{k=1, k \neq i}^{K} \mu_{k} \boldsymbol{R}_{i} \succeq \mathbf{0}, i=1, \ldots, K,
\end{aligned}
$$

where $\mu_{1}, \ldots, \mu_{K}$ are the dual variables associated with the trace inequality constraints of the inner problem of (9). Replacing the inner problem of (9) with its dual (13), we obtain the following problem

$$
\begin{aligned}
& \max _{\substack{\boldsymbol{V}_{i} \in \mathbb{H}^{N+1} \\
i=1, \ldots, K}}\left\{\begin{array}{c}
\max _{\substack{\mu_{i} \geq 0, i=1, \ldots, K}} \sum_{i=1}^{K} \mu_{i} \sigma_{i}^{2} \\
\text { s.t. } \boldsymbol{Y}_{i}\left(\mu_{1} \boldsymbol{V}_{1}, \ldots, \mu_{K} \boldsymbol{V}_{K}\right) \succeq \mathbf{0}, \\
i=1, \ldots, K,
\end{array}\right\} \\
& \text { s.t. } \operatorname{Tr}\left(\boldsymbol{V}_{i}\right) \leq\left(1+r_{i}^{2}\right), i=1, \ldots, K, \\
& {\left[\boldsymbol{V}_{i}\right]_{N_{t}+1}=1, i=1, \ldots, K, } \\
& \boldsymbol{V}_{1}, \ldots, \boldsymbol{V}_{K} \succeq \mathbf{0} .
\end{aligned}
$$

Since strong duality holds for the inner parts of (9) and (14), the two problems have the same optimal objective value. 
One may observe a connection between (11) and (14):

$$
\left[\boldsymbol{A}_{i}\right]_{N_{t}+1}=\mu_{i}, \quad \boldsymbol{A}_{i}=\mu_{i} \boldsymbol{V}_{i}, i=1, \ldots, K .
$$

In fact, (11) and (14) are equivalent problems, as we show in Appendix the following lemma:

Lemma 1 If $\left(\boldsymbol{A}_{1}^{\star}, \ldots, \boldsymbol{A}_{K}^{\star}\right)$ is an optimal solution of (11), then

$$
\begin{aligned}
\left(\boldsymbol{V}_{1}^{\star}, \ldots, \boldsymbol{V}_{K}^{\star}\right) & =\left(\boldsymbol{A}_{1}^{\star} /\left[\boldsymbol{A}_{1}^{\star}\right]_{N_{t}+1}, \ldots, \boldsymbol{A}_{K}^{\star} /\left[\boldsymbol{A}_{K}^{\star}\right]_{N_{t}+1}\right), \\
\left(\mu_{1}^{\star}, \ldots, \mu_{K}^{\star}\right) & =\left(\left[\boldsymbol{A}_{1}^{\star}\right]_{N_{t}+1}, \ldots,\left[\boldsymbol{A}_{K}^{\star}\right]_{N_{t}+1}\right)
\end{aligned}
$$

is an optimal outer-inner solution pair of (14). If $\left(\boldsymbol{V}_{1}^{\star}, \ldots, \boldsymbol{V}_{K}^{\star}, \mu_{1}^{\star}, \ldots, \mu_{K}^{\star}\right)$ is an optimal outer-inner solution of (14), then $\left(\boldsymbol{A}_{1}^{\star}, \ldots, \boldsymbol{A}_{K}^{\star}\right)=\left(\mu_{1}^{\star} \boldsymbol{V}_{1}^{\star}, \ldots, \mu_{K}^{\star} \boldsymbol{V}_{K}^{\star}\right)$ is optimal to $(11)$.

Lemma 1 shows that $\left(\boldsymbol{V}_{1}^{\star}, \ldots, \boldsymbol{V}_{K}^{\star}\right)$ of (14) only differs from $\left(\boldsymbol{A}_{1}^{\star}, \ldots, \boldsymbol{A}_{K}^{\star}\right)$ of (11) up to a positive scalar. Hence, (14) and (11) attain the same optimal objective value, implying that (9) and (6) attain the same optimal objective value. By Lemma 1, one can further show that $\left(\boldsymbol{W}_{1}^{\star}, \ldots, \boldsymbol{W}_{K}^{\star}\right)$, the optimal primal solution of (6), is also optimal to (9). The detailed proof is presented in Appendix.

\section{B. Implication and Concluding Remark}

To show that the robust SDR problem (6) has a rank-one solution, we still need to prove that the optimal $\left(\boldsymbol{W}_{1}, \ldots, \boldsymbol{W}_{K}\right)$ of (9) is also optimal to (6). Now, let us assume:

Condition 1 The optimal solution of the inner minimization of problem (9), $\left(\boldsymbol{W}_{1}^{\star}, \ldots, \boldsymbol{W}_{K}^{\star}\right)$, is unique.

Condition 1 is considered mild; by numerical experience, Condition 1 is found to hold all the time. Under Condition 1, we can infer from Fact 1 and Proposition 1 that the SDR problem (6) has a rank-one solution. Hence, we conclude that

Claim 1 Under Condition 1, the SDR problem (6) solves the worst-cast robust problem (4) optimally.

Our analysis above narrows down the SDR optimality question to the proof of unique rank-one solution of the inner minimization problem of (9). As a future research direction, it would be interesting to investigate sufficient conditions under which Condition 1 holds true.

\section{APPENDIX}

KKT conditions of (6)

The KKT conditions of (6) and (11) can be shown to be

$$
\begin{aligned}
& \boldsymbol{W}_{1}, \ldots, \boldsymbol{W}_{K} \succeq \mathbf{0}, \lambda_{1}, \ldots, \lambda_{K} \geq 0, \boldsymbol{A}_{1}, \ldots, \boldsymbol{A}_{K} \succeq \mathbf{0}, \\
& \boldsymbol{\Psi}_{i}\left(\boldsymbol{W}_{1}, \ldots, \boldsymbol{W}_{K}, \lambda_{i}\right) \succeq \mathbf{0}, i=1, \ldots, K \\
& \boldsymbol{Y}_{i}\left(\boldsymbol{A}_{1}, \ldots, \boldsymbol{A}_{K}\right) \succeq \mathbf{0}, i=1, \ldots, K, \\
& \boldsymbol{\Psi}_{i}\left(\boldsymbol{W}_{1}, \ldots, \boldsymbol{W}_{K}, \lambda_{i}\right) \boldsymbol{A}_{i}=\mathbf{0}, i=1, \ldots, K, \\
& \boldsymbol{Y}_{i}\left(\boldsymbol{A}_{1}, \ldots, \boldsymbol{A}_{K}\right) \boldsymbol{W}_{i}=\mathbf{0}, i=1, \ldots, K, \\
& \operatorname{Tr}\left(\boldsymbol{A}_{i}\right) \leq\left(1+r_{i}^{2}\right)\left[\boldsymbol{A}_{i}\right]_{N_{t}+1}, i=1, \ldots, K, \\
& \left(\operatorname{Tr}\left(\boldsymbol{A}_{i}\right)-\left(1+r_{i}^{2}\right)\left[\boldsymbol{A}_{i}\right]_{N_{t}+1}\right) \lambda_{i}=0, i=1, \ldots, K,
\end{aligned}
$$

where $\boldsymbol{\Psi}_{i}(\cdot)$ and $\boldsymbol{Y}_{i}(\cdot)$ are defined in (7) and (12), respectively. Proof of Lemma 1: Lemma 1 can be easily proved by inspection of (14) and (11). What remains is to show that $\mu_{i}^{\star}>0$ and $\left[\boldsymbol{A}_{i}^{\star}\right]_{N_{t}+1}>0$ for all $i=1, \ldots, K$. The former has been proved in [13, Proposition 4.2]; while the latter can be proved as follows. One can observe from (17a) and (17f) that $\left[\boldsymbol{A}_{i}^{\star}\right]_{N_{t}+1}=0$ results in $\boldsymbol{A}_{i}^{\star}=\mathbf{0}$. In this case, $\boldsymbol{Y}_{i}\left(\boldsymbol{A}_{1}^{\star}, \ldots, \boldsymbol{A}_{K}^{\star}\right)$ in (12) is positive definite, i.e., $\boldsymbol{Y}_{i}\left(\boldsymbol{A}_{1}^{\star}, \ldots, \boldsymbol{A}_{K}^{\star}\right) \succ \mathbf{0}$. By the complementary slackness (17e), this leads to the primal solution $\boldsymbol{W}_{i}^{\star}=\mathbf{0}$, which however violates (17b) [see (7)] due to $\sigma_{i}^{2}>0$.

Proof of Proposition 1: Here we prove that $\left(\boldsymbol{W}_{1}^{\star}, \ldots, \boldsymbol{W}_{K}^{\star}\right)$, the optimal primal solution of (6), is also optimal to (9). By Lemma 1 which shows that $\left(\boldsymbol{A}_{1}^{\star} /\left[\boldsymbol{A}_{1}^{\star}\right]_{N_{t}+1}\right.$, $\left.\ldots, \boldsymbol{A}_{K}^{\star} /\left[\boldsymbol{A}_{K}^{\star}\right]_{N_{t}+1}\right)$ is an optimal outer maximizer of (9), it suffices to show that $\left(\boldsymbol{W}_{1}^{\star}, \ldots, \boldsymbol{W}_{K}^{\star}\right)$ is optimal to the following problem

$$
\begin{aligned}
\min _{\boldsymbol{W}_{1}, \ldots, \boldsymbol{W}_{K} \succeq \mathbf{0}} & \sum_{i=1}^{K} \operatorname{Tr}\left(\boldsymbol{W}_{i}\right) \\
\text { s.t. } & \operatorname{Tr}\left(\left(\frac{1}{\gamma_{i}} \boldsymbol{W}_{i}-\sum_{k=1, k \neq i}^{K} \boldsymbol{W}_{k}\right)\left[\boldsymbol{I} \overline{\boldsymbol{h}}_{i}\right] \boldsymbol{A}_{i}^{\star}\left[\begin{array}{c}
\boldsymbol{I} \\
\overline{\boldsymbol{h}}_{i}^{H}
\end{array}\right]\right) \\
& \geq \sigma_{i}^{2}\left[\boldsymbol{A}_{i}^{\star}\right]_{N_{t}+1}, i=1, \ldots, K .
\end{aligned}
$$

This can be shown by examining that $\left(\boldsymbol{W}_{1}^{\star}, \ldots, \boldsymbol{W}_{K}^{\star}\right)$ satisfies the KKT conditions of (18), which are given as follows:

$\boldsymbol{W}_{1}, \ldots, \boldsymbol{W}_{K} \succeq \mathbf{0}, \mu_{1}, \ldots, \mu_{K} \geq 0$,

$\boldsymbol{Y}_{i}\left(\mu_{1}\left(\boldsymbol{A}_{1}^{\star} /\left[\boldsymbol{A}_{1}^{\star}\right]_{N_{t}+1}\right), \ldots, \mu_{K}\left(\boldsymbol{A}_{K}^{\star} /\left[\boldsymbol{A}_{K}^{\star}\right]_{N_{t}+1}\right)\right) \succeq \mathbf{0}$,

$\boldsymbol{Y}_{i}\left(\mu_{1}\left(\boldsymbol{A}_{1}^{\star} /\left[\boldsymbol{A}_{1}^{\star}\right]_{N_{t}+1}\right), \ldots, \mu_{K}\left(\boldsymbol{A}_{K}^{\star} /\left[\boldsymbol{A}_{K}^{\star}\right]_{N_{t}+1}\right)\right) \boldsymbol{W}_{i}=\mathbf{0}$

$\operatorname{Tr}\left(\left(\frac{1}{\gamma_{i}} \boldsymbol{W}_{i}-\sum_{k=1, k \neq i}^{K} \boldsymbol{W}_{k}\right)\left[\boldsymbol{I} \overline{\boldsymbol{h}}_{i}\right] \boldsymbol{A}_{i}^{\star}\left[\begin{array}{c}\boldsymbol{I} \\ \overline{\boldsymbol{h}}_{i}^{H}\end{array}\right]\right)=\sigma_{i}^{2}\left[\boldsymbol{A}_{i}^{\star}\right]_{N_{t}+1}$,

for $i=1, \ldots, K$.

Since $\left(\boldsymbol{W}_{1}^{\star}, \ldots, \boldsymbol{W}_{K}^{\star}, \lambda_{1}^{\star}, \ldots, \lambda_{K}^{\star}\right)$ and $\left(\boldsymbol{A}_{1}^{\star}, \ldots, \boldsymbol{A}_{K}^{\star}\right)$ satisfy the KKT conditions in (17a), (17c) and (17e), $\left(\boldsymbol{W}_{1}^{\star}, \ldots, \boldsymbol{W}_{K}^{\star}\right) \quad$ and $\left(\mu_{1}, \ldots, \mu_{K}\right) \quad \triangleq$ $\left(\left[\boldsymbol{A}_{1}^{\star}\right]_{N_{t}+1}, \ldots,\left[\boldsymbol{A}_{K}^{\star}\right]_{N_{t}+1}\right)$ satisfy (19a), (19b) and (19c). To show that $\left(\boldsymbol{W}_{1}^{\star}, \ldots, \boldsymbol{W}_{K}^{\star}\right)$ also fulfills (19d), let us consider an alternative representation of (6):

Lemma 2 Problem (6) can be equivalently expressed as the following problem

$$
\begin{aligned}
& \min _{\substack{\boldsymbol{W}_{i} \succeq \mathbf{0}, i=1, \ldots, K}} \sum_{i=1}^{K} \operatorname{Tr}\left(\boldsymbol{W}_{i}\right) \\
& \text { s.t. } \min _{\boldsymbol{V}_{i} \in \mathcal{V}_{i}} \operatorname{Tr}\left(\left(\frac{1}{\gamma_{i}} \boldsymbol{W}_{i}-\sum_{k=1, k \neq i}^{K} \boldsymbol{W}_{k}\right)\left[\boldsymbol{I} \overline{\boldsymbol{h}}_{i}\right] \boldsymbol{V}_{i}\left[\begin{array}{c}
\boldsymbol{I} \\
\overline{\boldsymbol{h}}_{i}^{H}
\end{array}\right]\right) \\
& \geq \sigma_{i}^{2}, i=1, \ldots, K,
\end{aligned}
$$


where $\mathcal{V}_{i}=\left\{\boldsymbol{V}_{i} \in \mathbb{H}^{N_{t}+1} \mid \operatorname{Tr}\left(\boldsymbol{V}_{i}\right) \leq\left(1+r_{i}^{2}\right),\left[\boldsymbol{V}_{i}\right]_{N_{t}+1}=\right.$ $\left.1, \boldsymbol{V}_{i} \succeq \mathbf{0}\right\}$.

It is easy to verity that, for $\left(\boldsymbol{W}_{1}^{\star}, \ldots, \boldsymbol{W}_{K}^{\star}\right)$,

$$
\begin{aligned}
\min _{\boldsymbol{V}_{i} \in \mathcal{V}_{i}} & \operatorname{Tr}\left(\left(\frac{1}{\gamma_{i}} \boldsymbol{W}_{i}^{\star}-\sum_{k=1, k \neq i}^{K} \boldsymbol{W}_{k}^{\star}\right)\left[\boldsymbol{I} \overline{\boldsymbol{h}}_{i}\right] \boldsymbol{V}_{i}\left[\begin{array}{c}
\boldsymbol{I} \\
\overline{\boldsymbol{h}}_{i}^{H}
\end{array}\right]\right) \\
= & \sigma_{i}^{2}, i=1, \ldots, K
\end{aligned}
$$

i.e., the inequality constraints in (20b) are all active for the optimal solution $\left(\boldsymbol{W}_{1}^{\star}, \ldots, \boldsymbol{W}_{K}^{\star}\right)$. Hence, to show that (19d) is also fulfilled by $\left(\boldsymbol{W}_{1}^{\star}, \ldots, \boldsymbol{W}_{K}^{\star}\right)$, it is sufficient to prove that

$$
\begin{aligned}
& \boldsymbol{A}_{i}^{\star} /\left[\boldsymbol{A}_{i}^{\star}\right]_{N_{t}+1}= \\
& \arg \min _{\boldsymbol{V}_{i} \succeq \mathbf{0}} \operatorname{Tr}\left(\left(\frac{1}{\gamma_{i}} \boldsymbol{W}_{i}^{\star}-\sum_{k=1, k \neq i}^{K} \boldsymbol{W}_{k}^{\star}\right)\left[\begin{array}{ll}
\boldsymbol{I} & \left.\overline{\boldsymbol{h}}_{i}\right]
\end{array}\right] \boldsymbol{V}_{i}\left[\begin{array}{c}
\boldsymbol{I} \\
\overline{\boldsymbol{h}}_{i}^{H}
\end{array}\right]\right) \\
& \text { s.t. } \operatorname{Tr}\left(\boldsymbol{V}_{i}\right) \leq\left(1+r_{i}^{2}\right) \text {, } \\
& {\left[\boldsymbol{V}_{i}\right]_{N_{t}+1}=1 \text {, }}
\end{aligned}
$$

for all $i=1, \ldots, K$. Let $\xi_{i}$ and $\tau_{i}$ be the dual variables associated with the constraints in (22a) and (22b), respectively, and define

$$
\begin{aligned}
& \tilde{\boldsymbol{\Psi}}_{i}\left(\boldsymbol{W}_{1}^{\star}, \ldots, \boldsymbol{W}_{K}^{\star}, \xi_{i}, \tau_{i}\right) \\
& \triangleq\left[\begin{array}{c}
\boldsymbol{I} \\
\overline{\boldsymbol{h}}_{i}^{H}
\end{array}\right]\left(\frac{1}{\gamma_{i}} \boldsymbol{W}_{i}^{\star}-\sum_{k=1, k \neq i}^{K} \boldsymbol{W}_{k}^{\star}\right)\left[\boldsymbol{I} \overline{\boldsymbol{h}}_{i}\right]+\left[\begin{array}{cc}
\xi_{i} \boldsymbol{I} & \mathbf{0} \\
\mathbf{0} & \xi_{i}+\tau_{i}
\end{array}\right] .
\end{aligned}
$$

The KKT conditions of the minimization problem in (22) can be obtained as

$$
\begin{aligned}
& \operatorname{Tr}\left(\boldsymbol{V}_{i}\right) \leq\left(1+r_{i}^{2}\right),\left[\boldsymbol{V}_{i}\right]_{N_{t}+1}=1, \boldsymbol{V}_{i} \succeq \mathbf{0}, \xi \geq 0, \tau_{i} \in \mathbb{R}, \\
& \tilde{\boldsymbol{\Psi}}_{i}\left(\boldsymbol{W}_{1}^{\star}, \ldots, \boldsymbol{W}_{K}^{\star}, \xi_{i}, \tau_{i}\right) \succeq \mathbf{0}, \\
& \tilde{\boldsymbol{\Psi}}_{i}\left(\boldsymbol{W}_{1}^{\star}, \ldots, \boldsymbol{W}_{K}^{\star}, \xi_{i}, \tau_{i}\right) \boldsymbol{V}_{i}=\mathbf{0}, \\
& \xi_{i}\left(\operatorname{Tr}\left(\boldsymbol{V}_{i}\right)-\left(1+r_{i}^{2}\right)\right)=0, \tau_{i}\left(\left[\boldsymbol{V}_{i}\right]_{N_{t}+1}-1\right)=0 .
\end{aligned}
$$

For each $i \in\{1, \ldots, K\}$, let $\boldsymbol{V}_{i}^{\star}=\boldsymbol{A}_{i}^{\star} /\left[\boldsymbol{A}_{i}^{\star}\right]_{N_{t}+1}, \xi_{i}^{\star}=$ $\lambda_{i}^{\star}, \quad \tau_{i}^{\star}=-\sigma_{i}^{2}-\left(1+r_{i}^{2}\right) \lambda_{i}^{\star}$. It follows from the KKT conditions in (17a), (17b), (17d) and (17g) that $\left(\boldsymbol{V}_{i}^{\star}, \xi_{i}^{\star}, \tau_{i}^{\star}\right)$ satisfies all the conditions in (23). Thus (22) is true for all $i=1, \ldots, K$. The proof is then completed.

Proof of Lemma 2: It suffices to show that (6b) is equivalent to $(20 \mathrm{~b})$. Note that (6b) is equivalent to

$$
\begin{aligned}
\min _{\left\|\boldsymbol{e}_{i}\right\|^{2} \leq r_{i}^{2}} & \left\{\left(\overline{\boldsymbol{h}}_{i}+\boldsymbol{e}_{i}\right)^{H}\left(\frac{1}{\gamma_{i}} \boldsymbol{W}_{i}-\sum_{k=1, k \neq i}^{K} \boldsymbol{W}_{k}\right)\left(\overline{\boldsymbol{h}}_{i}+\boldsymbol{e}_{i}\right)\right\} \\
& \geq \sigma_{i}^{2}, i=1, \ldots, K .
\end{aligned}
$$

(the equivalence is owing to the $\mathcal{S}$-Lemma; see [8], [11]). Note that the minimization problem on the left-hand side of (24) may not be convex with respect to $\left(\boldsymbol{e}_{1}, \ldots, \boldsymbol{e}_{K}\right)$ because the matrix $\left(\frac{1}{\gamma_{i}} \boldsymbol{W}_{i}-\sum_{k \neq i}^{K} \boldsymbol{W}_{k}\right)$ may not be positive semidefinite. Nevertheless, SDR can be applied. Through the same procedure as in obtaining (9), one can obtain the SDR problem of the minimization problem in (24) as

$\min _{\boldsymbol{V}_{i} \in \mathcal{V}_{i}} \operatorname{Tr}\left(\left(\frac{1}{\gamma_{i}} \boldsymbol{W}_{i}-\sum_{k=1, k \neq i}^{K} \boldsymbol{W}_{k}\right)\left[\boldsymbol{I} \overline{\boldsymbol{h}}_{i}\right] \boldsymbol{V}_{i}\left[\begin{array}{c}\boldsymbol{I} \\ \overline{\boldsymbol{h}}_{i}^{H}\end{array}\right]\right)$.

While (25) is obtained by relaxation of the rank of $\boldsymbol{V}_{i}$, the SDR problem (25) is actually tight and optimal to the minimization problem in (24); see [14, Lemma 3.1]. We thus obtain (20b) by substituting (25) into (24).

\section{ACKNOWLEDGEMEnTs}

This work is supported in part by National Science Council, R.O.C., under Grant NSC-99-2221-E-007-052-MY3, by a General Research Fund of Hong Kong Research Grant Council (CUHK 415908), and by a Direct Grant awarded by the Chinese University of Hong Kong (Project Code 2050489).

\section{REFERENCES}

[1] M. Bengtsson and B. Ottersten, "Optimal and suboptimal transmit beamforming," Chapter 18 in Handbook of Antennas in Wireless Communications, L. C. Godara, Ed., CRC Press, Aug. 2001.

[2] D. J. Love, R. W. Heath, V. K. N. Lau, D. Gesbert, B. D. Rao, and M. Andrews, "An overview of limited feedback in wireless communication systems," IEEE Journal on Sel. Areas in Comm., vol. 26, pp. 1341-1365, Oct. 2008.

[3] M. B. Shenouda and T. N. Davidson, "Probabilistically-constrained approaches to the design of the multiple antenna downlink," in Proc. IEEE Asilomar Conf. Signals, Systems and Computers, Pacific Grove, Oct. 26-29, 2008, pp. 1120-1124.

[4] K.-Y. Wang, A. M.-C. So, T.-H. Chang, W.-K. Ma, and C.-Y. Chi, "Outage constrained robust transmit optimization for multiuser MISO downlinks: Tractable approximations by conic optimization," submitted to IEEE Trans. Signal Process., 2011, abridged versions published in EUSIPCO 2010 and ICASSP 2011.

[5] M. B. Shenouda and T. N. Davidson, "Nonlinear and linear broadcasting with QoS requirements: Tractable approaches for bounded channel uncertainties," IEEE Trans. Signal Process., vol. 57, no. 5, pp. 19361947, May 2009.

[6] _ "Convex conic formulations of robust downlink precoder designs with quality of service constraints," IEEE J. Sel. Topics in Signal Process., vol. 1, pp. 714-724, Dec. 2007.

[7] N. Vučić and H. Boche, "Robust QoS-constrained optimization of downlink multiuser MISO systems," IEEE Trans. Signal Process., vol. 57, pp. 714-725, Feb. 2009.

[8] G. Zheng, K.-K. Wong, and T.-S. Ng, "Robust linear MIMO in the downlink: A worst-case optimization with ellipsoidal uncertainty regions," EURASIP Journal on Advances in Signal Process., vol. 2008, pp. 1-15, June 2008, Article ID 609028.

[9] Z.-Q. Luo and T.-H. Chang, "SDP relaxation of homogeneous quadratic optimization: Approximation bounds and applications," Chapter 4 in Convex Optimization in Signal Processing and Communications, D.P. Palomar and Y. Eldar, Eds., UK: Cambridge University, 2010.

[10] Z.-Q. Luo, W.-K. Ma, A. M.-C. So, Y. Ye, and S. Zhang, "Semidefinite relaxation of quadratic optimization problems," IEEE Signal Process. Mag., pp. 20-34, May 2010.

[11] E. Song, Q. Shi, M. Sanjabi, R. Sun, and Z.-Q. Luo, "Robust SINRconstrained MISO downlink beamforming: When is semidefinite programming relaxation tight?" in Proc. IEEE ICASSP, Prague, Czech, May 22-27, 2011, pp. 3096-2099.

[12] S. Boyd and L. Vandenberghe, Convex Optimization. Cambridge, UK Cambridge University Press, 2004.

[13] Y. Huang and D. P. Palomar, "A dual perspective on separable semidefinite programming with applications to optimal downlink beamforming," IEEE Trans. Signal Process., vol. 58, no. 8, pp. 4253-4271, August 2010.

[14] $\longrightarrow$, "Rank-constrained separable semidefinite program with applications to optimal beamforming," IEEE Trans. Signal Process., vol. 58, no. 2, pp. 664-678, Feb. 2010. 